\title{
LA MEDIACIÓN ECLESIÁSTICA EN LAS CORONACIONES DE BIZANCIO Y SUS IMPLICACIONES SIMBÓLICAS
}

\author{
JAUME AURELL \\ UNIVERSIDAD DE NAVARRA. España
}

Resumen: Las coronaciones en Bizancio se muestran como un extraordinario campo de experimentación, de entidad ritual y ceremonial, donde convergen buena parte de las ideas políticas relacionadas con origen divino de la soberanía de los emperadores. Este artículo analiza la intervención eclesiástica en la ceremonia de coronación imperial, la idea de "mediación" que esta realidad materializa, sus ricas implicaciones simbólicas y su relevancia al ser una costumbre extendida a todo el Occidente medieval.

Palabras clave: Coronación, Ritual, Mediación, Unción, Patriarca Constantinopla.

\section{THE ECCLESIASTICAL MEDIATION IN THE CORONATIONS OF THE BYZANTINE EMPEROR AND ITS SYMBOLIC IMPLICATIONS}

\begin{abstract}
Coronations in Byzantium were an extraordinary testing ground rich in ritual and ceremonial significance in which many of the political ideas related to the divine origin of the power of the emperors converged. This article engages the Church intervention in the ceremony of the imperial coronation, the idea of "mediation" that this reality embodies, its symbolic implications, and its relevance as the ceremony that was also spread in the European Western Kingdoms.
\end{abstract}

Palabras clave: Coronation, Simbology, Ritual, Mediation, Unction.

Recibido: 19.05.2016 - Aceptado: 23.08.2016

Correspondencia: Jaume Aurell.

Email: saurell@unav.es

Profesor Departamento de Historia.

Universidad de Navarra - 31080 - Pamplona (España). 


\section{T as coronaciones en Bizancio se muestran como un extraordinario campo de experimentación, de entidad ritual y ceremonial, donde convergen buena parte de las ideas políticas relacionadas} con origen divino de la soberanía de los emperadores ${ }^{1}$. La ceremonia de investidura del emperador se manifestó desde el principio como una de las formas más eficaces de hacer tangible esta realidad, particularmente a través de la fuerza visual del momento de la coronación ${ }^{2}$. Sin embargo, fue en Bizancio, paradójicamente, donde se introdujo la costumbre de que el obispo (en su caso, el patriarca de Constantinopla) ejerciera de ministro de la coronación. La intervención del patriarca en las ceremonias de inauguración de los emperadores en el siglo $\mathrm{V}$ está relacionada con el contexto de las herejías cristológicas de la época. De hecho, junto a esa intervención directa del patriarca en la coronación, se introduce también la costumbre del juramento de ortodoxia por parte del emperador, con el patriarca de testigo, lo que supuso un nuevo ámbito de influencia mayor de la jerarquía eclesiástica.

La introducción de la figura del patriarca como ministro de la coronación supuso una mengua, a nivel simbólico y a nivel visual, de la idea del origen divino de la soberanía del emperador, pues se añadía de hecho una mediación entre Jesucristo y el emperador a la hora de solemnizar el traspaso de poder.

Partiendo de estas premisas, este artículo está dedicado a un análisis comparativo de la realidad de la intervención eclesiástica en la ceremonia de la coronación imperial, dejando para una segunda parte el examen de la ficción de la coronación del emperador recibida directamente por Jesucristo, sus ángeles y sus santos, tal como aparece fijada en el motivo iconográfico de la coronación

$1 \quad$ Este artículo se ha realizado en el marco del grupo de investigación "Las formas de representación del poder en la Península Ibérica bajomedieval: ceremonias, juramentos y divisas» (ref. HAR2014-58542-P, 2015-2017), Ministerio de Economía y Competitividad del Gobierno de España, y del grupo "Religión y Sociedad Civil", del Instituto de Cultura y Sociedad (ICS), Universidad de Navarra. Quiero dejar constancia también de mi agradecimiento a José Marín Riveros, por sus valiosas sugerencias.

2 Las tres fuentes básicas para la ceremonia de inauguración de los emperadores bizantinos son: Jacob Goar, Euchologion sive Rituale Graecorum, Paris, 1647; Pseudo-Codinus, Treatise, ed. J. Verpeaux, Le traité des offices du Pseudo-Kodinus, Paris, 1966; y, por fin, el "Libro de las Ceremonias", comisionado por Constantino VII, con varias ediciones: Constantini Porphyrogeniti imperatoris, De cerimoniis aulae Byzantinae libri duo, ed. J. J. Reiske, 2 vols., Bonn 1829-30; Constantine Porphyrogenitus, Livre des Cérémonies, ed. A. Vogt, 2 vols., Paris, Société d'Édition Les Belles Lettres, 1967 (edición francesa parcial); The Book of Ceremonies, eds. Ann Moffat, Maxeme Tall, Camberra, Australian Association for Byzantine Studies, 2012, 2 vols. (edición inglesa parcial). 
celeste $^{3}$.Cada una de estas realidades implica una realidad histórica y simbólica respectivamente, lo que exige un tipo de tratamiento específico para cada una de ellas desde un punto de vista historiográfico, reflejadas la primera (la histórica) en este artículo y la segunda (la ficcional) en el artículo del número siguiente de la revista.

\section{La introducción de la mediación eclesiástica en la coronación imperial (siglo $\mathrm{V}$ )}

Si al reino Visigótico le corresponde la rehabilitación del rito de la unción hacia mediados del siglo VII, procedente de la tradición del reino bíblico de Israel, el imperio Bizantino fue el primero que introdujo la realidad de la coronación real por parte del obispo, en su caso el patriarca de Constantinopla, hacia mediados del siglo $\mathrm{V}^{4}$. Ambas realidades, unción y coronación por parte del obispo, fueron asumidas posteriormente por la mayor parte de las monarquías de Occidente a lo largo de la edad media. Estas dos variaciones en el rito, que enfatizaban el papel mediador de los eclesiásticos en la recepción del poder del soberano, se introdujeron en Bizancio (aunque la unción mucho más tardíamente que la coronación) porque la proclamación de un emperador no implicaba un ceremonial fijo, sino que asumía diferentes formas según la coyuntura política del momento, el modo de acceso del emperador al poder, o la propia personalidad del soberano que era investido ${ }^{5}$.

Aunque los autores difieren en este punto, parece que fue Marciano el primero que introdujo la costumbre de ser coronado por el patriarca en 450, tal como parece desprenderse de algunos testimonios externos pero fiables ${ }^{6}$.

3 André Grabar, L'empereur dans l'art byzantin, London, Variorum Reprints, 1936, 112-122; Andrea Torno Ginnasi, L'incoronazione celeste nel mondo bizantino. Politica, ceremoniale, numismatica e arti figurative, Oxford, Archaeopress, 2014. Ver también, Miguel Cortés, "El tema de la coronación simbólica en el arte bizantino de la edad de oro", Erytheia 9 (1988): 133-141.

4 Sobre la introducción en Bizancio de la figura del patriarca como ministro de la coronación: Wilhelm Ensslin, "Zur Frage nach der ersten Kaiserkrönung durch den Patriarchen und zur Bedeutung dieses Aktes im Wahlzeremoniell", Byzantinische Zeitschrift 42 (1943-49): 101-115. Sobre la introducción de la unción real en Bizancio: Chistopher Walter, "The Significance of unction in Bizantine icongraphy", Byzantine and Modern Greek Studies 2 (1976): 53-73; Gilbert Dagron, Emperor and Priest. The Imperial Office in Byzantium, Cambridge, Cambridge University Press, 2003, 267276; Miguel Arranz", "L'aspect rituel de l'onction des empereurs de Constantinople et (des tsars) de Moscou, Roma. Costantinopoli, Mosca. Da Roma alla terza Roma, Roma, La Sapienza, 1981, 407-15

Dagron, Emperor and Priest, 60.

Wilhelm Sickel fue el primero que documentó y discutió esta importante 
Marciano habría acudido al patriarca para legitimar su proclamación, la cual había sido puesta en duda al ser ejecutada sin el consentimiento el emperador reinante en Occidente, Valentiniano III. Marciano había violado el viejo principio constitucional que daba derecho al emperador en el trono a elegir a su colega en Oriente o a su sucesor en Occidente. Esta podría ser una razón plausible que habría hecho a Marciano acudir al patriarca, consiguiendo una legitimación por parte de una autoridad externa a él mismo, más cercana y respetada además por su autoridad espiritual y por su natural prestigio en el propio Bizancio al simbolizar, como obispo de la nea Roma, su tradición imperial. ${ }^{7}$ Todo ello habría sido favorecido también por la incesante divulgación de la creencia del origen divino de la autoridad imperial, una idea bien consolidada en Bizancio por la herencia helenística y su proximidad con Persia, lo que tendría lógicamente también una influencia en el ceremonial. ${ }^{8}$ Marciano mismo declaró en varias

realidad: Wilhelm Sickel, "Das byzantinische Krönungsrecht bis zum 10 . Jahrhundert", Byzantinische Zeitschrift 7 (1898): 511-557, especialmente 517 y 539; Friedhelm Winkelmann, "Zur Rolle der Patriarchen von Konstantinopel bei den Kaiserwechseln in frühbyzantinischer Zeit, "Klio 60 (1978): 467-481; Arthur E.R. Boak, "Imperial Coronation Ceremonies of the Fifth Centuries", Harvard Studies in Classical Philology XXX (1919): 37-47; Ernst Kantorowicz, Laudes Regiae. A Study in Liturgical Acllamations and Mediaeval Ruler Worship, Berkeley, University of California Press, 1958, 78-79; sin embargo, Frank E. Brightman, "Byzantine Imperial Coronations", Journal of Theological Studies 2 (1901): 359-392, especialmente p. 377, argumenta que la primera coronación con asistencia eclesiástica data de 602; Gilbert Dagron, por su parte, también manifiesta sus dudas, pues arguye que el patriarca no tuvo una significación real en la coronación hasta la coronación de Constancio II (Emperor and Prest, 80-82). Ver también Panayotis Yannopoulos, "Le Couronnment de l'empereur à Byzance: rituel et fond institutionnel", Byzantion 61 (1991): 71-91, especialmente 86-89; Peter Charanis, "The Imperial Crown Modiolus and Its Constitutional Significance", Byzantion, 12 (1937): 189-195; John B. Bury, "The Constitution of the Later Roman Empire", in Selected Essays, ed. H. Temperley, Cambridge, 1930, 103 y siguientes, donde arguye el posible origen persa de la intervención del patriarca en la ceremonia - que, en todo caso, no tendría el mismo significado, pues mientras el sumo sacerdote actuaba como representante de la religión en Persia, el patriarca constantinopolitano lo haría como representante del estado bizantino.

$7 \quad$ Francis Dvornik, Early Christian and Byzantine Political Philosophy, Washington, Dumbarton Oaks, 1966, II: 828. Ver también Dvornik, The Idea of Apostolicity in Byzantium and the Legend of the Apostle Andrew, Cambridge, Harvard University Press, 1958. Sobre el significado de la función del patriarca en la coronación imperial: Peter Charanis, "Imperial Coronation in Byzantium: Some New Evidence", Byzantina 8 (1976): 37-46.

8 Sobre la influencia del oriente Persa en el ceremonial bizantino, ver Héctor Herrera, "Simbología política del poder imperial en Bizancio: los pendientes de las coronas", en Herrera, Dimensiones de la Cultura Bizantina: Arte Poder y Legado 
ocasiones que él mismo había sido ascendido al trono no por el senado y el ejército sino también por Dios. Aunque la idea de Dios como fuente de potestad imperial no era nueva, ya que databa por lo menos del siglo III, Marciano fue el primer emperador en nombrar a Dios, el senado y el ejército conjuntamente como los tres elementos responsables de su elección. Mientras el senado y el ejército podían actuar por sí mismos, a través de representantes visibles, Dios debía ser representado por sus sacerdotes, por lo que la solución de la coronación por parte del patriarca tenía su sentido también desde esta perspectiva, así como por la mencionada del control de la ortodoxia por parte del patriarca. De este modo, se produce una conjunción de intereses políticos y religiosos: la legitimación del emperador ante su pueblo y la defensa de la fe por parte de la jerarquía ${ }^{9}$.

Otros autores sostienen que el primer emperador coronado por el patriarca fue León I en 457. Antes de esas fechas, sus predecesores habrían recibido la corona de un alto mando militar o un funcionario, según la tradición romana, siendo elevados sobre un escudo y aclamados por el ejército, el pueblo y el Senado, distinguiéndose en esa ceremonia los momentos y ceremonias de la proclamación, la aclamación y la coronación ${ }^{10}$.Sea uno u otro, en todo caso la coronación implica, a partir de Marciano y León I, un significado de consagración religiosa, un rito con fuertes connotaciones simbólicas. Es evidente también que la coronación por parte del patriarca es una "anomalía" respecto a la tradición romana, incluso entre los emperadores cristianos, y en cierta medida también respecto a la tradición persa.

A partir de Marciano y León I, la costumbre de la intervención del patriarca en la ceremonia de coronación del emperador quedó ya sólidamente fijada en Bizancio. De hecho, a lo largo de los mil años de su existencia, se han podido documentar únicamente tres excepciones a este modo tradicional de coronación. Constantino XI, el último emperador, fue coronado por un laico, pero de hecho nunca se hizo efectiva su proclamación como emperador. El usurpador Nicéforo Brienio se coronó a sí mismo. De todos modos, esta excepción no es demasiado significativa, pues Nicéforo nunca llegó a ser considerado oficialmente emperador porque su revuelta fracasó. Probablemente hubiera organizado una coronación "ortodoxa" de haber tenido éxito, porque el triunfo militar implicaba automáticamente legitimidad. Finalmente, otro

Histórico, Santiago de Chile, 1998, 395-438. [Publicado originalmente en Byzantion Nea Hellás 13-15, (1996): 15-53].

9 Peter Charanis, "Coronation and Its Constitutional Significance in the Later Roman Empire", Byzantion 15 (1940-41): 49-66, especialmente 53-54.

10 Louis Bréhier, Les institutions de l'empire byzantine, Paris, A. Michel, 1949. 
usurpador, Juan Cantacuzenus, anunció su revuelta poniéndose la corona en su cabeza con sus propias manos, pero cuando accedió realmente al trono, ansioso por conseguir el acuerdo con la Iglesia, fue coronado por el patriarca al modo tradicional. En este caso, la autocoronación asumió un significado claramente subversivo, pero en cuanto la situación política se estabilizó, el propio emperador se avino a una coronación en el modo tradicional, de manos del patriarca. Primero se hizo coronar por el patriarca de Jerusalén en Adrianópolis, por sus desavenencias con el titular de Constantinopla, que era partidario de Juan V Palaeologus y claramente hostil a Cantacuzenus. Pero incluso esta ceremonia fue considerada inadecuada por los partidarios de Cantacuzenus, ansiosos por hacer desaparecer cualquier duda acerca de la legitimidad de su autoridad, y finalmente el emperador se avino a ser coronado por el patriarca de Constantinopla una vez se verificó su predominio en la ciudad. Pero el anterior patriarca había sido removido y fue sustituido por Isidoro, que había sido nombrado precisamente por el nuevo emperador. Esta historia es muy expresiva de la situación a la que había llegado Imperio en el siglo XIV: Cantacuzenus y Isidore devinieron la fuente del poder de uno y otro: el primero propiciando la elección del segundo y el segundo coronando al primero ${ }^{11}$. En todo caso, todas estas excepciones confirman, por su propia naturaleza, el hecho de la importancia que asumió la intervención del patriarca en la investidura real. Si esta relevancia es de tipo simplemente simbólico o tiene consecuencias constitucionales es algo que la historiografía ha discutido mucho, aunque sin llegar a un acuerdo definitivo.

\section{La mediación simbólica del patriarca en las coronaciones}

El primer conjunto de coronaciones que conocemos con un cierto detalle son las practicadas desde mediados del siglo $\mathrm{V}$ a mediados del siglo VI. Cuando Constantino VII Porphyrogenitus, a mediados del siglo X, mandó compilar todo el material para la elaboración del "Libro de las Ceremonias" (De Caerimoniis aulae Byzantinae), en algún momento se decidió incorporar algunos pasajes redactados siglos atrás, alrededor de la época justinianea, por el maestro de ceremonias de Justiniano, Pedro el Patricio ${ }^{12}$.Por tanto, los relatos

11 Charanis, "Coronation", 54, 62-63; Steve Runciman en Journal of Hellenistic Studies LVIII (1938): 127 (book review).

12 Comentarios al Libro de Ceremonias, cuyas ediciones están citadas en la nota 1, en Jonh B. Bury, "The Ceremonial Book of Constantine Porphyrogennetos", The English Historical Review 22 (1907): 209-227; 417-39; Tsirpanlis, "The Imperial Coronation", 63-91; Louis Bréhier, Les institutions de l'empire byzantine, Paris, A. Michel, 1949; Arnold J. Toynbee, Constantine Porphyrogenitus and his World, 
que Pedro el Patricio realiza de las coronaciones de los emperadores del siglo $\mathrm{V}$ son bastante fiables, pues la cercanía cronológica es mucho mayor que las otras ceremonias consignadas en el "Libro de las ceremonias". Este tratado sitúa las coronaciones, quizás sorprendentemente, a la cabeza de la sección de las ceremonias civiles y de la promoción de los dignatarios, y por tanto la aleja deliberadamente de la sección de las ceremonias religiosas, a pesar del papel sustancial ejercido por el patriarca ${ }^{13}$. Distingue también entre una "vieja ceremonia", realizada durante el siglo V, que se inicia con una parada militar situada en la "Milestone" diecisiete de Constantinopla, el Hebdomon, y una "nueva ceremonia" (desde finales del siglo V), que se concentra en el Hipódromo y en la Iglesia de Santa Sofía ${ }^{14}$.

La primera coronación que recoge el "Libro de las ceremonias" es la de León I, proclamado emperador en el año 457. La sucesión de acontecimientos que se produjeron en ese ascenso al trono son ilustrativos de los diferentes actores que solían participar en este tipo de ceremonias. Después de su elección en el Senado, León fue proclamado emperador por aclamación por la multitud reunida en el Campo de Marte (Campus Martius). La proclamación popular se basaba en la legitimidad de los senadores, los militares y los oficiales de la corte, que eran los tres grupos a quienes correspondía la elección. León fue conducido entonces al tribunal donde Bousalgus, el compiductor - un oficial de alto rango -, colocó alrededor de su cuello una cadena militar (torques), mientras Olympius, otro compiductor, le colocaba otra cadena alrededor de su brazo derecho. Allí también asumió las ropas imperiales y se auto-impuso la diadema - un dato muy revelador, pues el emperador cedió esa prerrogativa al patriarca durante la parte religiosa de la ceremonia ${ }^{15}$. De hecho, Gilbert Dagron comenta que este es el verdadero momento de la coronación imperial - y que, por tanto, se trataría de una autocoronación en su sentido más estricto - más que la que tuvo lugar posteriormente en Santa Sofía de manos del patriarca, que sería más bien una confirmación del investidura imperial ${ }^{16}$.Armado con lanza y escudo, recibió el homenaje de los oficiales, según el orden de su rango. Entonces se dirigió al pueblo, refiriéndose a sí mismo con los títulos de Autocrator, Caesar, Victor y Augustus para siempre, nombrado por Dios y

\section{Oxford, Oxford University Press, 1973.}

13 Dagron, Emperor and Priest, 57.

14 Raymond Janin, Constantinople Byzantine. Dévelopment urbain et repertoire topographique, Paris, Archives de 1'Orient Chrétien, 1964, 446-9.

15 Boak, "Imperial Coronation", 38.

16 Dagron, Emperor and Priest, 63, 81. 
elegido por los soldados. La segunda parte de la ceremonia de su proclamación se produjo en Constantinopla, en la que visitó diversos lugares, la Iglesia de Santa Sofía entre ellas. Ahí depositó su corona en el altar, estando presente el patriarca Anatolio.

En este lacónico relato de la coronación de León I, los autores han discutido sobre si, en la segunda parte de la ceremonia, fue realmente el patriarca quien puso la corona sobre la cabeza del emperador - y por tanto, si él fue el primer emperador realmente coronado por el patriarca. Sin embargo, la mayor parte de ellos coinciden en la relevante circunstancia de que el emperador debió quitarse la corona al entrar en un lugar sacro. Y es precisamente en ese lugar sacro donde correspondía al patriarca volver a ponerle la corona para que pudiera abandonarla con la bendición de Dios. Edward Gibbon, mostrando cautela respecto al hecho de si realmente fue León I coronado por el patriarca pero en todo caso amparado por el hecho de la consolidación de esta costumbre en los inmediatos sucesores de León I, remarcó en su obra clásica la trascendencia histórica de este momento desde el punto de vista del ceremonial real ${ }^{17}$.Gibbon se basó en los testimonios de Teodoro el Lector y Tehophanes, que afirmaron que León fue coronado por el patriarca Anatolio. Sin embargo, el relato de Pedro el Patricio, compilado en el siglo X por el "Libro de las Ceremonias" de Constantino VII, refiere únicamente la presencia del patriarca en la ceremonia pero sin tomar parte activa. En todo caso, fuera o no efectivamente coronado por el patriarca, es evidente que a partir de la coronación de León I - y probablemente ya en la de su antecesor Marciano se introduce un elemento eclesiástico que ya no dejará de estar presente en la ceremonia de proclamación del emperador hasta la caída de Bizancio en 1453, e incluso tendrá su continuidad en la Rusia moderna y contemporánea de los zares.

Más allá del debate sobre la actuación del patriarca, también es importante destacar que en la coronación de León I se distingue claramente la parte militar de la coronación (que recoge muchos de los símbolos y las ceremonias más tradicionales, como la de Juliano el Apóstata en París en 360, que tan expresivamente narró Amiano Marcelino ${ }^{18}$ ) de la parte sacra. Esta distinción

$17 \quad$ "This appears to be the first origin of a ceremony which all the Christian princes of the world have since adopted, and from which the clergy have deduced the most formidable consequences" (Edward Gibbon, The decline and fall of the Roman Empire, London, Dent, 1993, capítulo 36).

18 Sobre el carácter germánico y militar de la coronación de Juliano, su influjo posterior, y la narración de Amiano, ver Ginnasi, L'incoronazione, 34-35. Ver también Brightman, "Byzantine Imperial", 365-366. 
se repite en la proclamación de Anastasio (491), aunque la ceremonia se realiza ya toda ella dentro de los muros de la ciudad de Constantinopla. Primero tuvo lugar la investidura civil y militar. Anastasio fue elevado en un escudo (gesto muy tradicional, de claras reminiscencias militares de origen germánico) y el campiductor le impuso el collar en el cuello, una costumbre en esta ocasión de origen celta. ${ }^{19}$ Después tuvo lugar la parte religiosa de la ceremonia, pero todavía fuera de la Iglesia: el emperador regresó al triklinos, donde el patriarca Eufemio, después de pronunciar un Kyrie eleison, le invistió con el chlamys (las ropas imperiales) y le impuso la corona en su cabeza. La presencia cada vez más activa del patriarca no implica todavía un reconocimiento institucional de la función de la Iglesia en la investidura del emperador, aunque ya se le asigna la tarea de garantizar la ortodoxia doctrinal del nuevo soberano. La verdadera coronación se produce todavía en un ámbito civil y militar, aunque ahora ya no se trata de una autocoronación como en el caso de León I sino de una coronación por parte del campiductor. Este esquema también se repitió en la proclamación de Justin I, quien fue coronado por el patriarca Juan II (519).

Las coronaciones de León I, Anastasio I y Justin I consolidaron el modelo de coronación del Autocrator, es decir, del emperador proclamado como único soberano. Junto a esa realidad, ya en el siglo $\mathrm{V}$ se fue desarrollando una ceremonia paralela, cuyos fundamentos se remontan al Imperio Romano tardoantiguo, y que más adelante sería tomada como modelo en Occidente por los reyes visigodos y posteriormente los francos y carolingios: la de quienes eran asociados al trono como co-emperadores, estando todavía en vida el emperador reinante ${ }^{20}$. Así sucedió, ya en el siglo $\mathrm{V}$, en la coronación de León II por León I (473) y de Zeno por León II. En estos casos, no era el patriarca quien imponía

19 El campiductor era un militar de alto rango: Boak, "Imperial Coronation", 38. Sobre la elevación del escudo, Héctor Herrera, "El significado del escudo en la Germania de Tácito", Anales de la Universidad Católica de Valparaíso, 4 (1957): 205-221.

20 Christopher Walter, "The Coronation of a Co-Emperor in the Skyllitzes Matritensis", Actes du XIVe congrès international des etudes Byzantines, eds. M. Berza y E. Stanescu, Bucarest, Editura Academici Republicii Socialiste Romania, 1975, 453-8.Respecto a la ceremonia de investidura real de los visigodos, José Orlandis, "La Iglesia visigótica y los problemas de la sucesión al trono en el siglo VII". Settimane di Studio del Centro italiano di studi sull'alto Medioevo, 7 (1960): 333-351. Respecto a la ceremonia de investidura en los carolingios, es probable que Carlomagno deseara una ceremonia civil (como hizo él mismo con su hijo Luis el Piadoso) y luego un simple bendición, pero el papa León III se le adelantó, y "creó" un rito inexistente en la tradición imperial, antecediendo la coronación religiosa a la civil (Janet L. Nelson, Politics and Ritual in Early Medieval Europe, London, Hambledon, 1986). 
la corona, sino el propio emperador - habitualmente, perteneciendo a la misma dinastía de quien era nombrado co-emperador. Esto sucedió por primera vez cuando León I cayó gravemente enfermo y decidió nombrar como co-emperador a su nieto León II, quien ya había sido proclamado previamente César. En este caso, la parte militar se realizó de la forma acostumbrada: el pueblo y los legados de diversas partes del Imperio se reunieron en el Hipódromo, donde los soldados llamaron al emperador. Éste apareció escoltado por los senadores, y fue requerido por la multitud para que coronara de co-emperador a su nieto. El César apareció en la escena, colocándose a la izquierda del Emperador, mientras el patriarca Acacio permaneció a su derecha y recitó una plegaria antes de la coronación - es bien significativo de la prelación del emperador que aparezca en el centro, con el patriarca a un lado. Entonces el emperador tomó la corona y la colocó en la cabeza del César, siendo proclamado a partir de ese momento como Augusto.

Respecto a este segundo grupo de proclamaciones, las de los coemperadores, el derecho de elección y captación provenía del propio emperador ya soberano. Esta costumbre provenía del bajo imperio, sin embargo en ningún momento se hizo automático que el hijo del emperador o cualquier otro miembro de su familia tuviera el derecho de ser nombrado soberano simplemente por razón de su nacimiento, sino sólo por el deseo del emperador ya reinante, hecho explícito en el acto de la nominación. En estos casos, parecía natural que la coronación fuera realizada por el emperador, en lugar del patriarca - que, en todo caso, ejercía de testigo cualificado. Este hecho confirma que la función del patriarca (incluso cuando, en el primer grupo de coronaciones, las del único soberano, Autocrator, él mismo imponía la corona) no tenía propiamente efectos jurídicos, por lo menos en los primeros siglos de existencia del imperio bizantino.

En el caso de la proclamación de un emperador cuando el trono está todavía vacante, el derecho de la nominación provenía de dos fuentes: los senadores y los militares. El pueblo de Constantinopla no tenía propiamente el derecho reconocido como agente de proclamación del emperador, pero estaba presente en la parte de la ceremonia que se tenía en el Hipódromo, reunido según distinción de colores (los Azules y los Verdes) como en las ocasiones en las que asistían a las carreras de caballos - una costumbre con mucha influencia por lo menos hasta la época de Justiniano ${ }^{21}$. Cuando el candidato era presentado al pueblo, éste podía aclamarlo o mostrar su desaprobación, pero no tenemos

21 Alan Cameron, Circus Factions. Blues and Greens in Rome and Byzantium, Oxford, Clarendon, 1976. 
base para suponer que el pueblo podía forzar un rechazo de la proclamación de un emperador una vez había sido nombrado por el senado y los militares. Quizás lo más propio sea decir que la fuente de autoridad del Emperador provenía de la elección de los senadores y los militares, y era sancionado por el pueblo. En realidad, y técnicamente hablando, a los emperadores los elige Dios, el ejército los proclama, el pueblo los aclama, el senado los nombra y eleva, y el patriarca los bendice. Por tanto, todos ellos no hacen sino reconocer la voluntad divina. El ceremonial, los gestos y los símbolos, incluso la escenificación del poder, se ponen al servicio de esa idea: el emperador es el "senescal" ("Hipóstrategos") de Dios, en frase gráfica de Pisides. En este contexto, el hecho de que el específico acto de la coronación fuera realizado por el patriarca suponía un reforzamiento simbólico de la soberanía del nuevo emperador, pero en ningún caso esa ceremonia tenía efectos propiamente constitucionales y jurídicos. De hecho, en el caso de los usurpadores, fue siempre el patriarca quien impuso la corona al emperador entrante, con la evidente intencionalidad de hacer ganar en legitimidad simbólica al nuevo soberano, pero siendo consciente de que no era un gesto suficiente para transmitir la soberanía.

El caso de la investidura de Basilio I (867-886), el fundador de la dinastía macedonia, representa una novedad importante, fundamentada por su condición de "soberano victorioso", más que por su condición de "heredero legítimo". Su investidura adquirió forma de "triunfo" más que de una coronación propiamente dicha, pues tuvo lugar tras su glorioso regreso a Constantinopla tras sus victorias en Tephrike y Germanikeia $(878-879)^{22}$. Este dato es importante para nuestra investigación, pues Basilio es el primero que introduce el tema de la "coronación celeste" en la iconografía bizantina, como veremos en la siguiente sección. Quizás Basilio incentivó este tipo de representaciones acuciado por el hecho de que no accedió al poder del modo tradicional y ortodoxo, basado en una sucesión hereditaria, sino a través del triunfo militar. De hecho, el tema de la herencia dinástica es una ficción que se basa en usos, prácticas y tradiciones, más que en normas constitucionales o leyes. Ya desde los isaúricos se intentó definir esencialmente y fijar jurídicamente de algún modo la idea dinástica, para que no entrara en colisión con la idea del emperador providencial ("pronooitikós"). Por tanto, técnicamente, en Bizancio funciona más bien una monarquía absoluta de carácter electivo, de ahí lo acuciante de la legitimación y justificación de cada emperador, aunque con las naturales diferencias a lo

22 Michael McCormick, Eternal Victory. Triumphal Rulership in Late Antiquity. Byzantium and the Early Medieval West, Cambridge, Cambridge University Press, 1986, 154-7 y 169. 
largo de toda su dilatada historia. Además, una vez asentado ya en el poder, y aprovechando todo su prestigio militar, Basilio se preocupó por organizar una segunda coronación, completando la que había recibido en 866 de manos de Miguel III, como co-emperador. Con esta segunda coronación, quizás Basilio trataba de superar su delito al asesinar a su propio protector Miguel III, a quien había tenido que quitar de en medio para acceder al poder. Pero esta segunda coronación debía realizarse "a la manera tradicional", tal como lo hacían los antiguos emperadores ${ }^{23}$.

Lo relevante de todos estos ejemplos para nuestra aproximación al tema es que, hasta finales del siglo $\mathrm{V}$, recogiendo la tradición bajo imperial iniciada durante la segunda mitad del siglo III, la parte esencial de la proclamación era la ceremonia militar, mientras que la segunda parte de la ceremonia, la que tenía lugar en el lugar sacro, era de importancia subsidiaria. Sin embargo, no se puede pasar por alto que, ya desde el principio, el emperador se quitaba la corona al entrar en el lugar sacro, lo que daba pie a que el patriarca se la volviera a imponer durante una ceremonia sacra, habitualmente al final de la santa misa. Esto generó, de modo natural, que cuando fuera desapareciendo esa primera parte de la ceremonia - la propiamente militar -, la función del patriarca, que era a quien correspondía indudablemente la coronación dentro del espacio sacro, fuera cobrando relevancia, tanto simbólica como constitucionalmente.

$23 \quad$ El dato de la "apelación a la tradición" de Basilio en su segunda coronación lo aporta sutilmente Dagron, Emperor et Priest, 74. Para el contexto de la compleja sucesión de Basilio I, ver Norman Tobias, Basil I Founder of the Macedonian Dynasty. A Study of the Political and Military History of the Byzantine Empire in the Ninth Century, Lewiston, The Edwin Mellen Press, 2007. 


\section{La intervención del patriarca: del significado simbólico al real}

En todo este proceso, se hace evidente en el creciente protagonismo del patriarca en la ceremonia de la proclamación. Su función evolucionó de un significado meramente simbólico hacia uno con mayores implicaciones jurídicas y constitucionales. En Occidente este maridaje entre Iglesia y Estado era más difícil que se produjera, teniendo presente la debilidad política de la ciudad de Roma. En Constantinopla, en cambio, la fortaleza política de la ciudad de Constantinopla fue paralela a la consolidación del patriarcado de Constantinopla frente a sus principales competidores, Antioquía y Alejandría, sobre todo a partir del concilio de Calcedonia (451) - significativamente, una fecha situada entre las coronaciones de Marciano y León I, los primeros emperadores que acudieron a la mediación del patriarca para su coronación.

Se entiende así mejor la progresiva relevancia del papel del patriarca de Constantinopla, el eclesiástico con mayor dignidad dentro del Imperio - que las fuentes refieren como Obispo más que como Arzobispo o Patriarca - como mediador entre Dios y la persona que, en adelante, gobernaría "por la gracia de Dios". Esto nunca implicó en Bizancio que el patriarca de Constantinopla tuviera una prelación sobre el emperador, como lo confirma el hecho de que correspondía al emperador imponer la corona cuando se investía a un coemperador, así como a la emperatriz. Pero la propia carga simbólica de estos ritos implicó, en la práctica, que el patriarca llegara a tener una autoridad muy grande de facto, sobre todo desde el momento en el que al emperador se le exigía una declaración explícita de fe cristiana, que correspondía al patriarca confirmar. Esto parece que sucedió por primera vez ya a finales del siglo V, en la coronación de Anastasio (491), pues el patriarca Eufemio requirió al nuevo emperador un juramento escrito en el que se comprometiera a mantener y defender la fe íntegra y no introducir novedades doctrinales en la santa Iglesia de $\operatorname{Dios}^{24}$.Aunque la petición de este juramento - que en el caso de Anastasio podría estar ocasionado por su reputación de heterodoxia - sólo se produjo de modo ocasional en los siguientes emperadores (Ciriaco lo pidió a Focas en 602 y Germano a León III en 716), esta costumbre se retomó ya definitivamente a partir del siglo IX, cuando Nicéforo lo pidió a Miguel I en 811, deviniendo una parte fija del ceremonial ${ }^{25}$.

$24 \quad$ Peter Charanis, Church and State in the Later Roman Empire: The Religious Policy of Anastasius I, Madison, The University of Wisconsin Press, 1939.

25 Charanis, “Coronation", 57-60; Brightman, "Byzantine Imperial”, 374. 


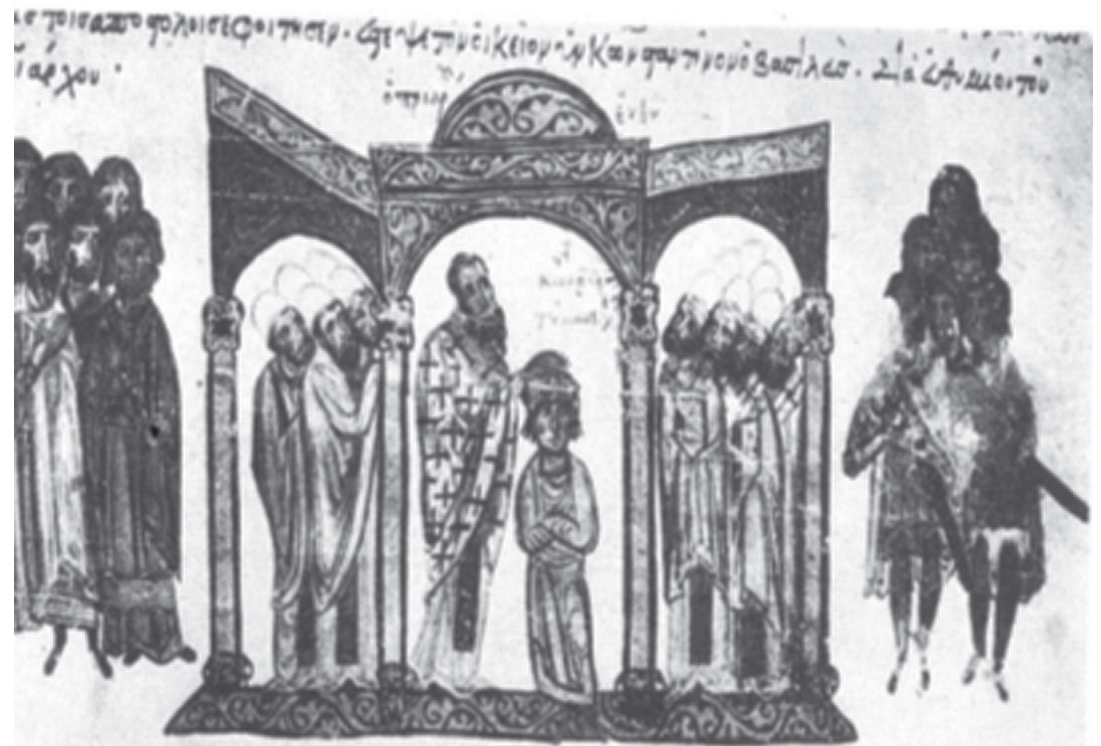

Fig. 1. Constantino VII coronado por el patriarca de Constantinopla. Codex Grcecus Matritensis Ioannis Skyllitzes, Crónica de Juan Skylitzes. Italia bizantina, siglos XI-XII, Synopsis of Histories by John Skylitze, Biblioteca Nacional de España manuscrito Graecus Vitr. 26-2 - Codex Grcecus, Madrid.

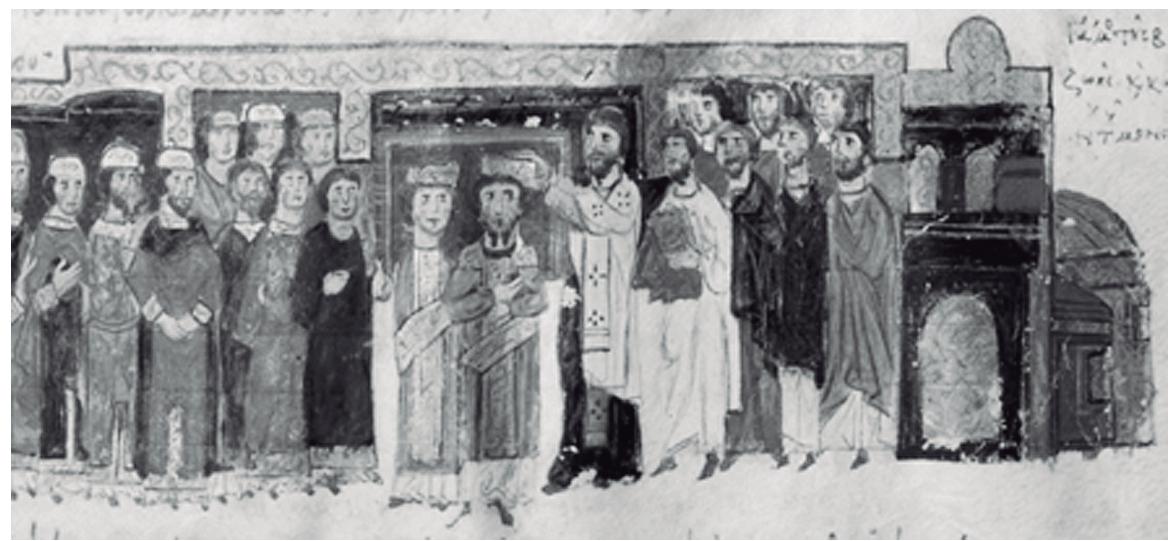

Fig. 2. Matrimonio y coronación de Constantino IX y Zoe en la basílica de Basilio

I. Codex Gracus Matritensis Ioannis Skyllitzes, Crónica de Juan Skylitzes. Italia Bizantina, siglos XI-XII, Biblioteca Nacional de España, manuscrito Graecus Vitr. 26-2 - Codex Grcecus, Madrid, España, f. 222v escena inferior. 
A raíz de estos datos, los historiadores se han preguntado si la participación del patriarca en la coronación de los emperadores bizantinos tenía sólo una significación religiosa y espiritual o implicaba también cambios jurídicos y constitucionales. Wilhelm Sickel fue el primero que se planteó a fondo la cuestión, y llegó a la conclusión de que la introducción del patriarca en la ceremonia de la coronación nunca tuvo efectos jurídicos. Al imponer la corona, el patriarca actuaba simplemente como representante de los electores - los magistrados, los senadores y los militares - y no estaba legitimado como fuente de autoridad. Por tanto, la coronación no tenía tampoco ninguna significación eclesiástica y el emperador podía prescindir de ella si así lo deseaba ${ }^{26}$. Para bizantinistas de la escuela alemana, la figura del emperador Marciano es esencial en esta cuestión, pues él fue quien confirió el privilegio de la coronación a los patriarcas, y por tanto el emperador mismo podía retirarlo. En esta línea, Steven Runciman concluyó que aunque la intervención del patriarca en la ceremonia de la coronación llegó a tener en ocasiones una gran relevancia, sobre todo cuando se dudaba de la capacidad moral del candidato, en realidad el patriarca era política y constitucionalmente siervo del emperador $^{27}$. Sin embargo, poco tiempo después algunos autores influyentes como John B. Bury, M. Manojlovic, Peter Charanis, y André Grabar postularon que la decisiva participación del patriarca en la ceremonia introdujo el nuevo principio constitucional de que la profesión de fe cristiana era una condición indispensable para asumir el oficio imperial e implicaba que el nuevo emperador había sido no sólo elegido por el ejército, el senado y el pueblo, sino también aceptado por la Iglesia ${ }^{28}$. Peter Charanis sostiene que la idea de que mientras que en el Imperio Romano cristianizado la idea de que la Iglesia era la única

$26 \quad$ Sickel, "Das byzantinische Krönungsrecht", 511-557. La tesis de Sickel fue seguida por Boak, "Imperial Coronation", 37-47, especialmente 46 y siguientes, aunque reconoce que el patriarca era la persona lógica para representar el símbolo que indicaba que el emperador gobernaba "por la gracia de Dios". Otros autores alemanes que siguieron a Sickel fueron Franz Dölger, Otto Treitinger y Wilhelm Ensslin y Ernst Stein. Ver Georg Ostrogorski y Ernst Stein, "Die Kroenungsordnungen des Zeremonienbuches. Chronologische und Verfassungsgeschichtliche Bemerkungen", Byzantion 7 (1932) : 185-233.

27 Steve Runciman, The Byzantine Theocracy, Cincinatti, The Weil Lectures, 2004, and, more especifically, his comment in the review, Steve Runciman in Journal of Hellenistic Studies LVIII (1938): 127.

28 John B. Bury, History of the Eastern Roman Empire from the Fall of Irene to the Accession of Basil I, London, Macmillan, 1912; M. Manojlovic, "Le people de Constantinople", Byzantion 11 (1936), 617-716; Peter Charanis, "Coronation and Its Constitutional Significance in the Later Roman Empire", Byzantion 15 (1940-41): 49-66. 
fuente de soberanía nunca acabó de implantarse, en cambio el juramento de fe requerido por los patriarcas en Constantinopla a los emperadores implicó una mayor relevancia constitucional de la Iglesia, que incluso podía llegar a tener derecho de veto en el caso de los emperadores heterodoxos ${ }^{29}$.

Más allá de su diferente interpretación, todos estos autores están de acuerdo con la idea bizantina de que el emperador recibe el poder de Cristo como es confirmado por la iconografía de la "coronación celeste" - y que la coronación del emperador por el patriarca representa el sacramento por el que el soberano queda conectado con Dios. Sin embargo, difieren en el grado de necesidad de la coronación por parte del patriarca. Este debate es relevante para el tema de las autocoronaciones, puesto que se trata de ver hasta qué punto el emperador era capaz de superar el carácter mediador de los eclesiásticos - en este caso representados por el patriarca de Constantinopla. A mí me parece que lo más relevante en este debate es la progresiva implantación y consolidación de un gesto ceremonial que tiene unos inicios titubeantes, que se convierte con el tiempo en una costumbre, y que llega finalmente a tener carácter de ley. Más que una postura esencialista, me parece que es preciso argüir por una postura historicista: si los emperadores de los siglos V al VIII valoraban la intervención del patriarca en términos más simbólicos que constitucionales, a partir del siglo IX, significativamente en el momento en el que se desarrolla la iconografía de la coronación del emperador por parte Cristo, la función mediadora del patriarca no es sólo simbólica sino que tiene carácter de necesidad. Así, si la función del patriarca en la coronación conoce una evolución de lo simbólico a lo real, la función de Jesucristo en la coronación conoce una evolución de lo simplemente teórico a lo iconográfico. Parece que el emperador siente la necesidad de enfatizar la intervención de Cristo, creando una iconografía específica a tal efecto, justo en el momento en el que se consolidan los efectos reales de la intervención del patriarca y en el momento en el que finaliza el decisivo interludio iconoclasta ${ }^{30}$.

Los testimonios de los contemporáneos bizantinos ponen de manifiesto el incremento de la dimensión sacra de la ceremonia de la coronación imperial $^{31}$. Cuando, a mediados del siglo $\mathrm{V}$, el patriarca empezó a intervenir

$29 \quad$ Charanis, "Coronation", 59.

30 Para la época preiconoclasta, especialmente para la dependencia inicial de la iconografía religiosa de la imperial antigua, ver el libro de Ernst Kitzinger, Byzantine Art in the Making, London, Faber and Faber, 1977.

31 Constantini Porphirogeniti De Cerimoniis Aulae Byzantinae, ed. Johan J. Reiske, Bonn, 1829-30, I: 191 y siguientes.; Jacob Goar, Euchologion sive Rituale 
en la ceremonia, los elementos militares y seculares no sufrieron ninguna transformación, e incluso en muchas ocasiones la coronación no tenía lugar en un lugar sacro. Pero con el paso del tiempo los elementos militares y seculares remitieron, dejando paso a una ceremonia plenamente religiosa, habitualmente celebrada en la iglesia de Santa Sofía. Además, el hecho de que el patriarca no interviniera en las coronaciones de los co-emperadores y de las conjugues del emperador pero estuviera presente y rezara las oraciones previstas, es una prueba más de su función de garante de facto, aunque su presencia y actuación no tuvieran consecuencias jurídicas e institucionales sino más bien simbólicas. No hay que olvidar tampoco, tal como lo ha analizado Panayotis Yannopoulos, los paralelismos que existen entre la ceremonia de la coronación imperial y la ceremonia de la ordenación de los obispos, lo que enfatizaría todavía más esta dimensión religiosa a la que me refiero ${ }^{32}$.

Algunos autores han relacionado la introducción de la costumbre de la coronación del emperador por el patriarca con la tradición persa en la que, después de algunos siglos en los que la autocoronación había dominado el ceremonial, se habían decantado en la época contemporánea a Marciano y León I por la coronación por parte del sumo sacerdote de los magianes. La misma diadema había sido importada de Persia, a través de la tradición helenística, por lo que pensar en estos intercambios simbólicos no es ni mucho menos descabellado. Ambos sacerdotes actuaban como representantes de su religión pero también de su estado ${ }^{33}$.

La evolución de la función del patriarca en las coronaciones imperiales, y el incremento de la dimensión sacra de esta ceremonia, es acorde con la idea de que la autoridad del emperador bizantino proveniente de Dios. Desde los tiempos de Julián el Apóstata (siglo IV) las ceremonias de coronación habían tenido un carácter claramente militar tanto en Roma como en Bizancio. Hacia el siglo XII, Manuel I consideró que su coronación, realizada en las montañas

Graecorum, Paris, 1647, 925 y siguientes; John Cantacuzenus, Historiae, ed. J. Schopen, Bonn, 1828-32, I: 196 y siguientes.; Pseudo-Codinus Curopalates, De Officis, ed. Immanuel Bekker, Bonn, 1839, 86 y siguientes; Symeon Bishop of Thessalonica, De Sacro Templo, en Migne, Patrologia Graegca CLV, Paris, 1866, 352 y siguientes.

32 Yannopoulos, "Le Couronnment de l'empereur", 71-91.

33 Sickel, "Das byzantinische Krönungsrecht, 511-577; John B. Bury, The Constitution of the Later Roman Empire, Cambridge, Cambridge University Press, 1910, 11 y siguientes; Constantine N. Tsirpanlis, "The Imperial Coronation and Theory in "De Ceremoniis Aulae Byzantinae" of Constantine VII Porphyrogennitus", Kleronomia 4 (1972): 63-91. 
de Cilicia por su padre Juan II e incluso con el acuerdo del ejército, no había sido suficiente, y quiso ser coronado de nuevo emperador por el patriarca en Constantinopla (1143). La idea de Dios como fuente de poder imperial encontró su mejor expresión en la dimensión religiosa de la ceremonia de la coronación. El patriarca no ejerce una función política, sino más bien sacerdotal, pues confiere la consagración al emperador y realiza la función de instrumento de la voluntad divina. Esta idea de "consagración" contribuye a explicar la expresión utilizada por León III de que el soberano en Bizancio es a la vez "sacerdote y emperador" - teniendo presente de que se trata de una fuente indirecta al propio Bizancio ${ }^{34}$. La amenaza para la Iglesia es clara, pero al mismo tiempo esta fórmula pone de manifiesto el fondo institucional de la idea de que el emperador es también el jefe supremo de los cristianos, aunque no en términos sacerdotales y de jerarquía sino más ministeriales.

\section{Referencias bibliográficas}

ARRANZ, MIGUEL(1981). “L'aspect rituel de l'onction des empereurs de Constantinople et (des tsars) de Moscou“, Roma. Costantinopoli, Mosca. Da Roma alla terza Roma. Roma: La Sapienza, 407-15.

BOAK, ARTHUR E.R. (1919). "Imperial Coronation Ceremonies of the Fifth Centuries," Harvard Studies in Classical Philology, 30, 37-47.

BRÉHIER, LOUIS (1949). Les institutions de l'empire byzantine. Paris: A. Michel.

BRIGHTMAN, FRANK E. (1901). "Byzantine Imperial Coronations," Journal of Theological Studies, 2, 359-392.

BURY, JONH B. (1907). "The Ceremonial Book of Constantine Porphyrogennetos", The English Historical Review, 22, 209-227.

BURY, JOHN B. (1910). The Constitution of the Later Roman Empire. Cambridge : Cambridge University Press.

BURY, JOHN B. (1912). History of the Eastern Roman Empire from the Fall of Irene to the Accession of Basil I. London: Macmillan.

BURY, JOHN B. (1930). "The Constitution of the Later Roman Empire", en Selected Essays, ed. H. Temperley. Cambridge: Cambrdige University Press.

CAMERON, ALAN (1976). Circus Factions. Blues and Greens in Rome and Byzantium. Oxford: Clarendon.

CHARANIS, PETER (1937). "The Imperial Crown Modiolus and Its Constitutional Significance”, Byzantion, 12, 189-195.

$34 \quad$ A esta dualidad del emperador dedicó una magnífica monografía Dagron, Emperor and Priest. Ver también George P. Majeska, "The Emperor in His Church: Imperial Ritual in the Church of St. Sophia", Byzantine Court Culture from 829 to 1204, ed. Henri Maguire, Washington, Dumbarton Oaks, 1997, 1-11. 
CHARANIS, PETER (1939). Church and State in the Later Roman Empire: The Religious Policy of Anastasius I. Madison: The University of Wisconsin Press.

CHARANIS, PETER (1940-1941). "Coronation and Its Constitutional Significance in the Later Roman Empire", Byzantion, 15, 49-66.

CHARANIS, PETER (1976). "Imperial Coronation in Byzantium: Some New Evidence", Byzantina, 8, 37-46.

CORTÉS, MIGUEL (1988). "El tema de la coronación simbólica en el arte bizantino de la edad de oro", Erytheia 9, 133-141.

CONSTANTINE PORPHYROGENITUS(1967). Livre des Cérémonies, ed. A. Vogt, 2 vols. Paris : Société d'Édition «Les Belles Lettres».

-------, (2012). The Book of Ceremonies, eds. Ann Moffat, Maxeme Tall. Camberra: Australian Association for Byzantine Studies.

DAGRON, GILBERT (2003). Emperor and Priest. The Imperial Office in Byzantium. Cambridge: Cambridge University Press.

DVORNIK, FRANCIS (1966). Early Christian and Byzantine Political Philosophy. Washington: Dumbarton Oaks

DVORNIK, FRANCIS (1958). The Idea of Apostolicity in Byzantium and the Legend of the Apostle Andrew. Cambridge: Harvard University Press.

ENSSLIN WILHELM (1943). "Zur Frage nach der ersten Kaiserkrönung durch den Patriarchen und zur Bedeutung dieses Aktes im Wahlzeremoniell”, Byzantinische Zeitschrift, 42, 101-115.

GIBBON, EDWARD (1993). The decline and fall of the Roman Empire. London: Dent. GINNASI, ANDREA Torno (2014). L'incoronazione celeste nel mondo bizantino. Politica, ceremoniale, numismatica e arti figurative. Oxford: Archaeopress.

GOAR, JACOB (1647). Euchologion sive Rituale Graecorum. Paris.

GRABAR, ANDRE (1936). L'empereur dans l'art byzantin. London: Variorum Reprints.

HERRERA, HÉCTOR (1957). "El significado del escudo en la Germania de Tácito". Anales de la Universidad Católica de Valparaíso, 4, 205-221.

, (1993-1996), "Simbología política del poder imperial en Bizancio: los pendientes de las coronas", Byzantion Nea Hellás 13-15, 15-53.

JANIN, RAYMOND(1964). Constantinople Byzantine. Dévelopment urbain et repertoire topographique. París: Archives de l'Orient Chrétien.

JOHN CANTACUZENUS (1929-32). Historiae, ed. J. Schopen. Bonn.

KANTOROWICZ, ERNST (1958). Laudes Regiae. A Study in Liturgical Acllamations and Mediaeval Ruler Worship, Berkeley: University of California Press.

KITZINGER, ENRST (1977). Byzantine Art in the Making, London: Faber and Faber. MANOJLOVIC, M. (1936). "Le people de Constantinople", Byzantion, 11, 617-716.

MCCORMICK, MICHAEL (1986). Eternal Victory. Triumphal Rulership in Late Antiquity. Byzantium and the Early Medieval West. Cambridge: Cambridge University Press.

MAJESKA, GEORGE P. (1997). "The Emperor in His Church: Imperial Ritual in the Church of St. Sophia”, Byzantine Court Culture from 829 to 1204, ed. Henri Maguire. Washington: Dumbarton Oaks, 1-11. 
NELSON, JANET L. (1986). Politics and Ritual in Early Medieval Europe.London: Hambledon.

ORLANDIS, JOSÉ (1960). "La Iglesia visigótica y los problemas de la sucesión al trono en el siglo VII". Settimane di Studio del Centro italiano di studi sull'alto Medioevo, 7, 333-351.

OSTROGORSKI, GEORG Y ERNST STEIN (1932). "Die Kroenungsordnungen des Zeremonienbuches. Chronologische und Verfassungsgeschichtliche Bemerkungen", Byzantion, 7. 185-233.

PSEUDO-CODINUS (1966). Treatise, ed. J. Verpeaux, Le traité des offices du PseudoKodinus. Paris.

RUNCIMAN, STEVE (2004). The Byzantine Theocracy, Cincinatti: The Weil Lectures.

SICKEL, WILHELM (1898). "Das byzantinische Krönungsrecht bis zum 10. Jahrhundert", Byzantinische Zeitschrift, 7, 511-557.

SYMEON BISHOP OF THESSALONICA (1866). De Sacro Templo, en Migne, Patrologia Graegca CLV, Paris.

TOBIAS, NORMAN (2007). Basil I Founder of the Macedonian Dynasty. A Study of the Political and Military History of the Byzantine Empire in the Ninth Century. Lewiston: The Edwin Mellen Press.

TOYNBEE, ARNOLD J. (1973).Constantine Porphyrogenitus and his World. Oxford: Oxford University Press.

TSIRPANLIS, CONSTANTINE N. (1972). "The Imperial Coronation and Theory in "De Ceremoniis Aulae Byzantinae" of Constantine VII Porphyrogennitus", Kleronomia, 4, 63-91.

WALTER, CHRISTOPHER (1975). "The Coronation of a Co-Emperor in the Skyllitzes Matritensis," Actes du XIVe congrès international des etudes Byzantines, eds. M. Berza y E. Stanescu. Bucarest: Editura Academici Republicii Socialiste Romania, 453-458.

WALTER, CHRISTOPHER (1976). "The Significance of unction in Bizantine icongraphy", Byzantine and Modern Greek Studies, 2, 53-73.

WINKELMANN, FRIEDHELM (1978). "Zur Rolle der Patriarchen von Konstantinopel bei den Kaiserwechseln in frühbyzantinischer Zeit", Klio 60, 467-481.

YANNOPOULOS, PANAYOTIS (1991). "Le Couronnment de l'empereur à Byzance: rituel et fond institutionnel”, Byzantion, 61, 71-91. 